\title{
Fitotoxicidad de herbicidas postemergentes sobre Adesmia bicolor (Poir.) DC y control de malezas asociadas
}

\author{
Autrán, V.A.; E.C. Puricelli y J.A. Andrés
}

\begin{abstract}
RESUMEN
Un mejor conocimiento del manejo de las especies nativas de leguminosas forrajeras como Adesmia bicolor puede ayudar a lograr una mayor calidad y estabilidad en la producción de los pastizales. No existe información disponible en la Argentina sobre el tipo y dosis de herbicidas adecuados para el control de malezas en A. bicolor. Los objetivos fueron evaluar el efecto fitotóxico de herbicidas postemergentes a diferentes dosis sobre A. bicolor y el control de las malezas latifoliadas que crecen asociadas en ensayos a campo durante 2009 y 2010. El diseño fue de parcelas divididas donde la parcela mayor fue el tipo de herbicida (2,4-DB e imazetapir) y la parcela menor la dosis (1/4X, 1/2X, $1 \mathrm{X}, 2 \mathrm{X}, 4 \mathrm{X}, 8 \mathrm{X}$, siendo $\mathrm{X}=250 \mathrm{~g}$ i.a. ha-1 para 2,4-DB y $\mathrm{X}=100 \mathrm{~g}$ i.a. ha-1 para imazetapir), con tres repeticiones para cada tratamiento. La materia seca de A. bicolor y el control de malezas se ajustaron a curvas de dosis-respuesta. Se observó efectos fitotóxicos aun con muy baja dosis de 2,4-DB, mientras que imazetapir mostró baja fitotoxicidad para las dosis entre 25 y $100 \mathrm{~g}$ a.i. ha ${ }^{-1}$. El control de malezas con ambos herbicidas fue mayor al $80 \%$ con dosis de $1 \mathrm{X}$.
\end{abstract}

Palabras clave: malezas latifoliadas, imazetapir, 2,4-DB.

Autrán, V.A.; E.C. Puricelli and J.A. Andrés, 2013. Postemergence herbicide phytotoxicity on Adesmia bicolor (Poir.) DC and associated weed control. Agriscientia 30 (2): 57-67

\section{SUMMARY}

A better knowledge of the management of native legume species such as Adesmia bicolor will help to achieve higher quality and stability of grassland production. In Argentina, there is no information about herbicides types and adequate doses for A. bicolor. In 2009 and 2010, field trials were conducted to evaluate the phytotoxic effect of different doses of herbicides on $A$. bicolor and the control of broad-leaved weed species associated with the crop. The experimental design was a split-plot with 3 replicates. The main plot consisted of the type of herbicide (2,4-DB and imazethapyr) and the subplot of the different doses (1/4X, 1/2X, 1X, 2X, 4X, 8X, being $X=250 \mathrm{~g}$ a.i. ha-1 for 2,4-DB and $X=100 \mathrm{~g}$ a.i. ha-1 for imazethapyr) with three repetitions for each treatment. The dry matter production of $A$. bicolor and weed control fitted to a dose- 
response curve. All doses of 2,4-DB showed phytotoxic effects on A. bicolor while imazethapyr showed little injury between 25 and $100 \mathrm{~g}$ a.i. ha-1 doses. Weed control with both herbicides was $80 \%$ with $1 \mathrm{X}$.

Key words: broad-leaved weeds, imazethapyr, 2,4-DB

V.A. Autrán y J.A. Andrés: Facultad Agronomía y Veterinaria, Universidad Nacional de Río Cuarto, Ruta Nac. 36 - Km. 601, X5804 BYA Río Cuarto, Córdoba, Argentina. E.C. Puricelli: Facultad de Ciencias Agrarias, Universidad Nacional de Rosario CC 14, S2125 ZAA ZavaIla, Santa Fe, Argentina. Correspondencia a: valeautran@gmail.com

\section{INTRODUCCIÓN}

El avance de la frontera agrícola ha modificado el paisaje de los ecosistemas áridos y semiáridos en la Argentina, y la ganadería se ha desplazado en parte a zonas con grandes limitantes edafológicas, climáticas e hídricas; por lo tanto, el área remanente de pastizales naturales tiene un uso más intensivo por el aumento de la carga animal e incrementa el porcentaje de suelo desnudo (Demaría et al., 2008). Así, en los últimos años, el área de pastizales naturales de la región árida-semiárida central y subhúmeda seca argentina se redujo aproximadamente un 35\% debido a su reemplazo por cultivos agrícolas o por forrajeras anuales y perennes (Aguilera et al., 1998).

En ese marco, la introducción de especies nativas de leguminosas forrajeras tiene como objetivo lograr una mayor calidad y producción sostenible de forraje durante todo el año (Sanabria et al., 1995; Ovalle et al., 2005; Canobas et al., 2006; Zalazar et al., 2009). El contenido de proteínas de las leguminosas es generalmente mayor que el de las gramíneas, por lo que permiten mejorar el valor nutritivo de la dieta animal. Además, las leguminosas, incrementan la fertilidad del suelo debido a su capacidad de fijar nitrógeno, facilitando de esta manera su disponibilidad a las gramíneas acompañantes (Castillo Gallegosa 2005; Ovalle et al., 2005; Rojas Hernández et al., 2005). Asimismo, el mantenimiento de la condición ecológica de los pastizales naturales es muy importante ya que son fuente de biodiversidad y conservan la estabilidad de estos ambientes muy susceptibles a la degradación.

Entre las leguminosas nativas, Adesmia bicolor
(Poir.) DC. es una especie perenne de ciclo otoño invernal ampliamente distribuida como un componente menor en los pastizales en Argentina, Brasil y Uruguay (Scheffer-Basso et al., 2002; Rebuffo et al., 2006). Esta especie posee buenos atributos nutricionales para el ganado y ofrece buenas posibilidades para la introducción y domesticación en los sistemas productivos de las regiones semiáridas (Dodd \& Orr, 1995; Sanabria et al., 1995; Scheffer-Basso et al., 2000, 2001; Bianco 2002). La especie se adapta a temperaturas extremas $(-15$ ${ }^{\circ} \mathrm{C}$ ), tolera bien la sequía y puede crecer aun en altitudes superiores a 1700 metros sobre el nivel del mar (Coll \& Zarza, 1992; Veneciano et al., 2005). Actualmente se la está investigando para introducirla al agroecosistema con el fin de utilizarla como pastura pura o consociada con otras especies forrajeras (Traverso et al., 2005; Autrán \& Puricelli, 2010; Vileta et al., 2010).

Uno de los problemas que presentan las especies nativas es la alta infestación de malezas latifoliadas en el período de establecimiento ya que poseen un crecimiento lento inicial y son menos competitivas (Pinzón et al., 1985, 1989; Rivas Pantoja et al., 2009). El uso de herbicidas contribuye a controlar malezas pero también pueden tener efecto fitotóxico sobre los cultivos (Altman \& Rovira, 1989; Dissanayake et al., 1998), produciendo alteraciones en la fisiología de la planta (Carson et al., 1991; Smiley \& Wilkins, 1992). El daño puede ser tanto en tejidos aéreos como subterráneos y en ocasiones suele llegar a ser letal para el cultivo (Hess, 1993). Así, el control químico de malezas puede ser una técnica eficaz y económica si el herbicida es totalmente selectivo para la planta forrajera, de modo que no presente efectos de fitotoxicidad que perjudiquen el desarrollo o su rendimiento (Verzig- 
nassi et al., 2005; Rivas Pantoja et al., 2009). Es sabido que el control químico de malezas es una herramienta habitual en otras especies cultivadas leguminosas como soja (Glycine max L.) (Young et al., 2003), alfalfa (Medicago sativa L.) (Wilson, 1981; Tonks et al., 1991) y trébol rojo (Trifolium pratense L.) (Ceballos et al., 2005), aunque en todos esos cultivos es esencial utilizar la dosis adecuada de herbicida que permita un eficiente control de malezas sin producir fitotoxicidad.

En general, la información disponible para el tipo y dosis de herbicidas empleados en el manejo de malezas en pasturas de especies nativas es muy escasa y está basada en prácticas procedentes de otras especies de leguminosas provenientes de otros países (Renzi et al., 2006). En particular en A. bicolor no existe información sobre control químico de malezas. Entre los herbicidas postemergentes usados para el control de malezas en otras especies de leguminosas y citados en algunos casos como selectivos se encuentran imazetapir (Darwent et al., 1997) y 2,4-DB (Cudney et al., 1993), con dispares resultados en relación a fitotoxicidad.

El objetivo de este estudio fue evaluar a campo el efecto fitotóxico de un amplio rango de dosis de los herbicidas 2,4-DB e imazetapir sobre A. bicolor y el control de las malezas latifoliadas asociadas.

\section{MATERIALES Y MÉTODOS}

El estudio se realizó en 2009 y 2010 en el campo experimental de la Facultad de Agronomía y Veterinaria de la Universidad Nacional de Río Cuarto, Córdoba (Argentina), localizado a 33 06' 23 latitud sur y $64^{\circ} 17^{\prime} 54$ longitud oeste sobre un suelo caracterizado como Hapludol típico con 2,3\% de materia orgánica y un $\mathrm{pH}$ de 6,8 , con $14,17 \%$ de arcilla, $41,97 \%$ de limo y $43,9 \%$ de arena. Las precipitaciones registradas en promedio mensual para el período de ocupación del ensayo fueron 51 $\mathrm{mm}$ para la primera fecha y $72 \mathrm{~mm}$ para la segunda fecha. Estos niveles de precipitación permitieron un adecuado estado de las plantas al momento de la aplicación. Los registros climáticos pertenecen a la estación meteorológica propia del campo experimental.

El área experimental fue laboreada con rastra de doble acción en febrero de 2008 y a los 20 días se aplicó glifosato (1200 g i.a. ha-1 4 48\%) como un barbecho químico. Se produjeron plantines de $A$. bicolor a partir de semillas y estolones portadores de ápices y, al menos, una hoja completamente expandida, correspondientes a la población de esta especie que crece en Villa Rumipal (Córdoba) (Veneciano et al., 2005). Estos se dejaron en macetas durante un mes con el fin de lograr buen tamaño de las plantas antes de ser llevadas al campo, donde en marzo de 2008 fueron trasplantados a una distancia de $50 \mathrm{~cm}$ entre sí y $50 \mathrm{~cm}$ entre las filas de la parcela experimental y se esperó hasta que toda la parcela estuviese cubierta. Para la segunda fecha ya se había logrado la sistematización para la obtención en gran escala de los plantines, lo que agilizó la implantación, procedimiento que se realizó en octubre de 2009 cuando se terminaron de obtener los datos de la primera aplicación; esto se hizo con el objeto, además, de probar otra fecha de implantación posiblemente más favorable que la anterior ya que siendo una especie nativa ésta se desconoce.

El diseño fue de parcelas divididas con tres repeticiones para cada tratamiento, siendo la parcela mayor de $1080 \mathrm{~m}^{2}$ con dos niveles de herbicida: $2,4-\mathrm{DB}(100 \%)$ e imazetapir (10\%) y la parcela menor de $25,7 \mathrm{~m}^{2}$ cada una; la dosis con siete niveles: $0 \mathrm{X}, 1 / 4 \mathrm{X}, 1 / 2 \mathrm{X}, 1 \mathrm{X}, 2 \mathrm{X}, 4 \mathrm{X}, 8 \mathrm{X}$, siendo $\mathrm{X}$ para 2, 4-DB $=250 \mathrm{~g}$ i.a. ha ${ }^{-1}$ y para imazetapir $X=100 \mathrm{~g}$ a.i. ha ${ }^{-1}$. Las fechas de aplicación fueron 14 de agosto de 2009 y 30 de marzo de 2010, sobre plantas de $A$. bicolor en estado vegetativo, con condiciones óptimas, adecuada humedad del suelo y relativa del aire, temperaturas templadas y a primera hora de la mañana. Se aplicaron los herbicidas en dos momentos distintos del ciclo cada año, ya que se desconoce la época óptima de aplicación de herbicidas y se decidió hacerlos en dos épocas contrastantes. Las aplicaciones se realizaron con una mochila de dióxido de carbono a presión constante. Se utilizaron boquillas Teejet 8002, a una presión de 270 kPa para asperjar un caudal de $180 \mathrm{~L} \mathrm{ha}^{-1}$.

El control de gramíneas se realizó con cletodim (24\%) con $120 \mathrm{~g}$ i.a. ha-1. Este herbicida no es fitotóxico aun en dosis mayores a las recomendadas en las especies de hoja ancha. Por otro lado, fue aplicado 20 días antes que 2,4-DB e imazetapir.

\section{Fitotoxicidad de imazetapir y 2,4-DB}

La fitotoxicidad se evalúo a través de la determinación del peso seco $\left(\mathrm{g} \mathrm{m}^{-2}\right)$ de A. bicolor 30 días después de la aplicación (DDA) y por la escala Rochecouste (Chaila, 1986) a los 7, 21 y 30 DDA (Tabla 1). Para la determinación de peso seco se tomaron tres muestras por parcela con un aro de $0,25 \mathrm{~m}^{2}$ arrojado al azar y el material vegetal recolectado se secó en una estufa a $55^{\circ} \mathrm{C}$ durante 7 días y se pesó en una balanza analítica. 
Tabla 1. Escala Rochecouste para evaluación de fitotoxicidad a los 7 días después de la aplicación (DDA), 21 DDA y 30 DDA.

\begin{tabular}{lc}
\hline Escala de Fitotoxicidad de Rochecouste & \\
\hline Efectos & Puntaje \\
Ningún efecto visible & 0 \\
Leve acción cáustica en hojas & 1 \\
Hojas cloróticas & 2 \\
Moderada acción cáustica en hojas & 3 \\
Moderada acción cáustica en hojas y tallos & 4 \\
Ligero daño en hojas y tallos & 5 \\
Ligero daño, muerte en brotes jóvenes & 6 \\
Tallos muertos 25\% & 7 \\
Tallos muertos 50\% & 8 \\
Tallos muertos 75\% & 9 \\
Muerte total & 10 \\
\hline
\end{tabular}

Se realizaron regresiones entre dosis y peso seco y entre dosis y la escala de Rochecouste utilizando el modelo log-logístico mediante el procedimiento NLIN del software estadístico SAS (Seefeldt et al., 1995) cuya expresión matemática es:

$$
\mathrm{y}=\mathrm{C}+\left((\mathrm{D}-\mathrm{C}) /\left(1+\left(\mathrm{x} / \mathrm{I}_{50}\right)^{\mathrm{b}}\right)\right)
$$

donde $y$ representa el peso seco o el porcentaje de control de malezas, $C$ es la respuesta promedio a altas dosis de herbicida, $D$ es la respuesta promedio a dosis cero de herbicida, $I_{50}$ es la dosis de herbicida que produce un $50 \%$ de disminución en el peso seco o en el porcentaje de control de malezas, $b$ es la pendiente de la curva en $I_{50}$ y $x$ es la dosis de herbicida.

Cuando los datos no ajustaron al modelo logístico se realizaron ajustes al modelo más adecuado (lineal o logarítmico).

\section{Control de malezas de imazetapir y 2,4-DB en A. bicolor}

Se realizaron muestreos de las malezas latifoliadas presentes utilizando un aro de $0,25 \mathrm{~m}^{2}$ arrojado al azar en cada subparcela un mes antes de cada aplicación. Se evaluó: densidad; abundancia, a) número de individuos, b) porcentaje del número de individuos sobre el total c) escala de BraunBlanquet (Chaila, 1986): apreciación del número de individuos de una especie estimada en forma cuantitativa visual; frecuencia de malezas, porcentaje del número de veces que la maleza está en cada subparcela muestreada sobre el total de muestreos y cobertura, a) como superficie de suelo que cubre la proyección una planta en porcentaje b) como grado de cobertura según escala de Braun-Blanquet.

El porcentaje de control de malezas fue poste- riormente evaluado a los 30 días de la aplicación de los herbicidas donde se evaluó por cada repetición: \% de cobertura y especies de malezas que se escaparon al control, utilizando de referencia la escala de clasificación de uso internacional E.W.R.C. (Consejo Europeo de Investigación de Malezas) (Chaila, 1986) adaptada y modificada (Artuzi \& Contiero, 2006); además, se ajustó a la curva logística mencionada para fitotoxicidad.

Los análisis estadísticos se realizaron utilizando el programa InfoStat versión 2010 (Di Rienzo et al., 2010).

\section{RESULTADOS Y DISCUSIÓN}

\section{Fitotoxicidad de imazetapir sobre A. bicolor}

En el ensayo con imazetapir el ANOVA del peso seco de A. bicolor detectó una interacción significativa entre los factores año, herbicida y dosis $(p=0,034)$. Por este motivo se realizaron regresiones separadas para cada año. La fitoxicidad de la dosis $1 X$ es mínima, valor en promedio de las repeticiones 3,8 en la escala Rochecouste. Si bien se observa cierto daño (Figura 1) muestra una disminución del $8 \%$ de producción de materia seca con respecto al testigo.

Se ha observado fitotoxicidad variable en leguminosas por herbicidas imidazolinonas (Eberlein \& Gutieri, 1994). Las especies forrajeras leguminosas muestran distinto grado de tolerancia a imazetapir. Así, Leucaena leucocephala es una especie nativa que con una dosis de $100 \mathrm{~g}$ i.a. ha-1 presentó un $18 \%$ de fitotoxicidad (Rivas Pantoja et al., 2009). Vicia villosa muestra una fitotoxicidad de 4 en la escala E.W.R.C. con 50 g i.a. ha-1 de imazetapir (Renzi, 2007). En Stylosantes capitata y S. macrocephala se encontró bajo nivel de fitotoxicidad a la dosis de $100 \mathrm{~g}$ i.a. ha-1 (Verzignassi et al., 2005). En Phaseolus vulgaris, Ríos y Pérez (1990) y Castro (1993) observaron porcentajes menores a 10\% de fitotoxicidad con dosis de 75 a 150 g i.a. ha-1, mientras que Cano y López (1996) hallaron extremada fitotoxicidad usando imazetapir en aplicaciones de 157 g i.a. ha-1

Los síntomas de fitotoxicidad se acrecentaron con el paso del tiempo. A los 7 DDA las dosis 1/4X, 1/2X, $1 X$ no superaron la escala 1 de Rochecouste. A los 21 y 30 DDA todas las dosis superiores a 100 g i.a ha-1 fueron fitotóxicas (Tabla 2). 


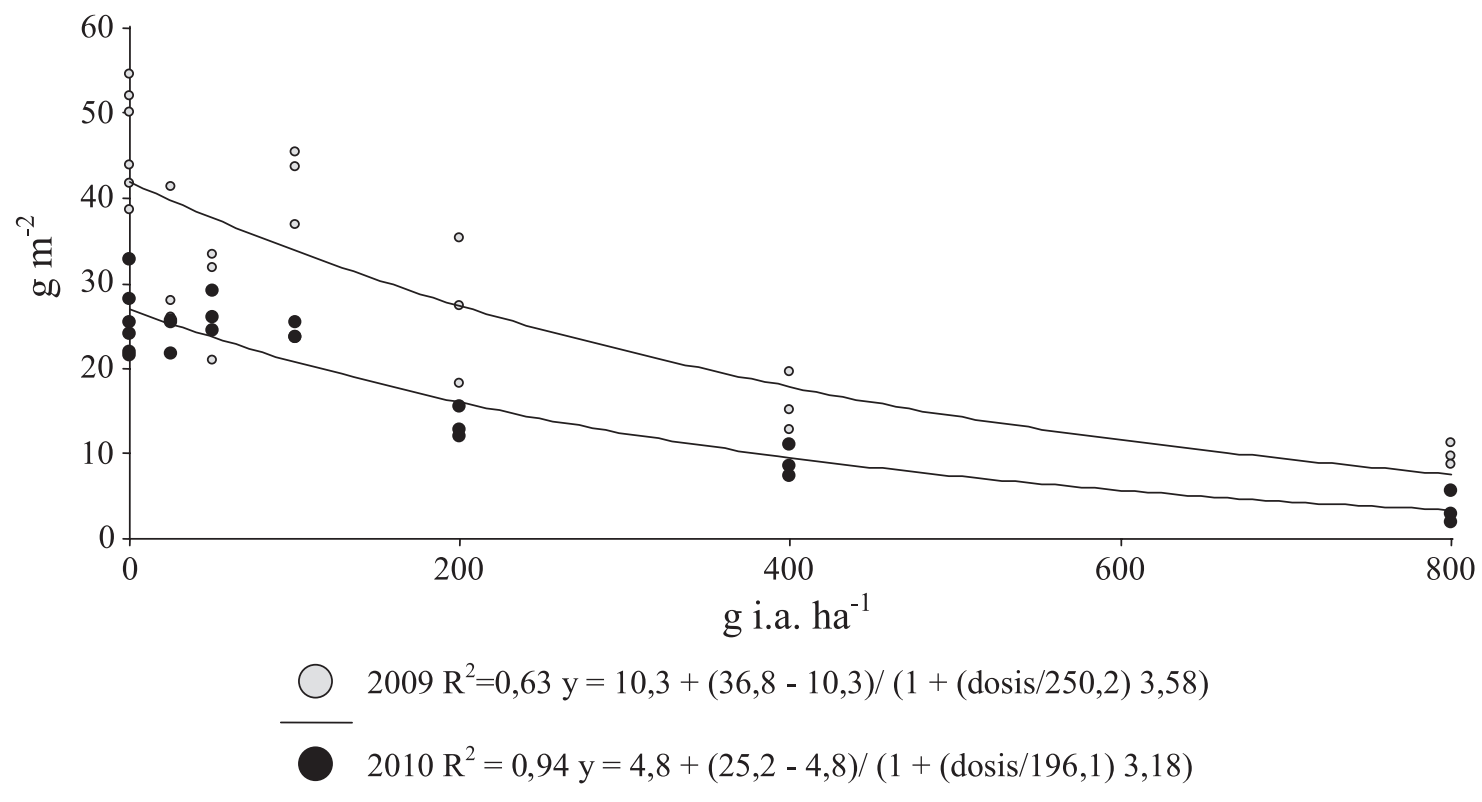

Figura 1. Efecto de diferentes dosis de imazetapir (g i.a. ha-1) sobre el peso seco $\left(\mathrm{g} \cdot \mathrm{m}^{-2}\right)$ de A. bicolor. $2009 \mathrm{R}^{2}=0,63 \mathrm{y}=10,3+(36,8-$ $10,3) /\left(1+(\text { dosis } / 250,2)^{3,58}\right) ; 2010 R^{2}=0,94 y=4,8+(25,2-4,8) /\left(1+(\text { dosis } / 196,1)^{3,18}\right)$.

\section{Fitotoxicidad de 2,4-DB sobre A. bicolor}

Se observó muy alta fitotoxicidad ya que la biomasa de $A$. bicolor se redujo significativamente aun con la dosis más baja utilizada del herbicida 2,4-DB. Estos datos impidieron obtener regresiones significativas, por lo cual en la Figura 2 se muestran sólo las medias de peso seco para cada dosis en cada año. En otros estudios 2,4-DB está recomendado en leguminosas. Así, Istilart (2003) observó fitotoxicidad intermedia con este herbicida en Vicia villosa a la dosis de 800 a $1600 \mathrm{~g}$ i.a. ha $^{-1}$; Conrad \& Stritzke (1980) informaron que en Trifolium vesiculosum 2,4DB a 1200 g i.a. ha-1 es selectivo y controla efectivamente malezas asociadas. En trabajos con otros herbicidas hormonales se encontraron variables efectos fitotóxicos según la especie de leguminosa, etapa o tamaño del cul-

Tabla 2. Fitotoxicidad de diferentes dosis de 2,4-DB e imazetapir a los 7 días después de la aplicación (DDA), 21 DDA y 30 DDA.

\begin{tabular}{|c|c|c|c|c|c|c|c|}
\hline Tratamiento & Dosis g.i.a.ha-1 & $\begin{array}{c}\text { Fitotoxicidad } 7 \\
\text { días DDA }\end{array}$ & E.E. & $\begin{array}{c}\text { Fitotoxicidad } 21 \\
\text { días DDA }\end{array}$ & E.E. & $\begin{array}{l}\text { Fitotoxicidad } \\
30 \text { días DDA }\end{array}$ & E.E. \\
\hline \multirow[t]{7}{*}{ 2,4-DB } & 2000 & 4 & $\pm 0,31 a$ & 10 & $\pm 0,39 a$ & 10 & $\pm 0,3 \mathrm{a}$ \\
\hline & 1000 & 3,67 & $\pm 0,31 a$ & 10 & $\pm 0,39 a$ & 10 & $\pm 0,3 \mathrm{a}$ \\
\hline & 500 & 2 & $\pm 0,31 b$ & 9,67 & $\pm 0,39 a$ & 10 & $\pm 0,3 \mathrm{a}$ \\
\hline & 250 & 1 & $\pm 0,31 \mathrm{c}$ & 9 & $\pm 0,39 a$ & 10 & $\pm 0,3 \mathrm{a}$ \\
\hline & 125 & 0,5 & $\pm 0,38 c$ & 8,5 & $\pm 0,68 \mathrm{a}$ & 10 & $\pm 0,52 a$ \\
\hline & 62,5 & 0,33 & $\pm 0,31 \mathrm{c}$ & 7,33 & $\pm 0,39 b$ & 10 & $\pm 0,37 \mathrm{a}$ \\
\hline & 0 & 0 & $\pm 0,31 \mathrm{c}$ & 0 & $\pm 0,39 e$ & 0 & $\pm 0,3 \mathrm{~g}$ \\
\hline \multirow[t]{7}{*}{ IMAZETAPIR } & 800 & 1 & $\pm 0,31 \mathrm{c}$ & 7 & $\pm 0,39 b$ & 8,67 & $\pm 0,3 \mathrm{~b}$ \\
\hline & 400 & 1 & $\pm 0,31 \mathrm{c}$ & 5 & $\pm 0,39 c$ & 6,67 & $\pm 0,3 \mathrm{c}$ \\
\hline & 200 & 0,33 & $\pm 0,31 \mathrm{c}$ & 3 & $\pm 0,39 d$ & 5,33 & $\pm 0,3 d$ \\
\hline & 100 & 0,33 & $\pm 0,31 \mathrm{c}$ & 2 & $\pm 0,39 d$ & 4 & $\pm 0,3 e$ \\
\hline & 50 & 0,33 & $\pm 0,31 \mathrm{c}$ & 1 & $\pm 0,39 d$ & 1,67 & $\pm 0,3 f$ \\
\hline & 25 & 0 & $\pm 0,31 \mathrm{c}$ & 0,67 & $\pm 0.39 \mathrm{e}$ & 0,67 & $\pm 0,3 \mathrm{~g}$ \\
\hline & 0 & 0 & $\pm 0,31 \mathrm{c}$ & 0 & $\pm 0,39 \mathrm{e}$ & 0 & $\pm 0,3 \mathrm{~g}$ \\
\hline
\end{tabular}




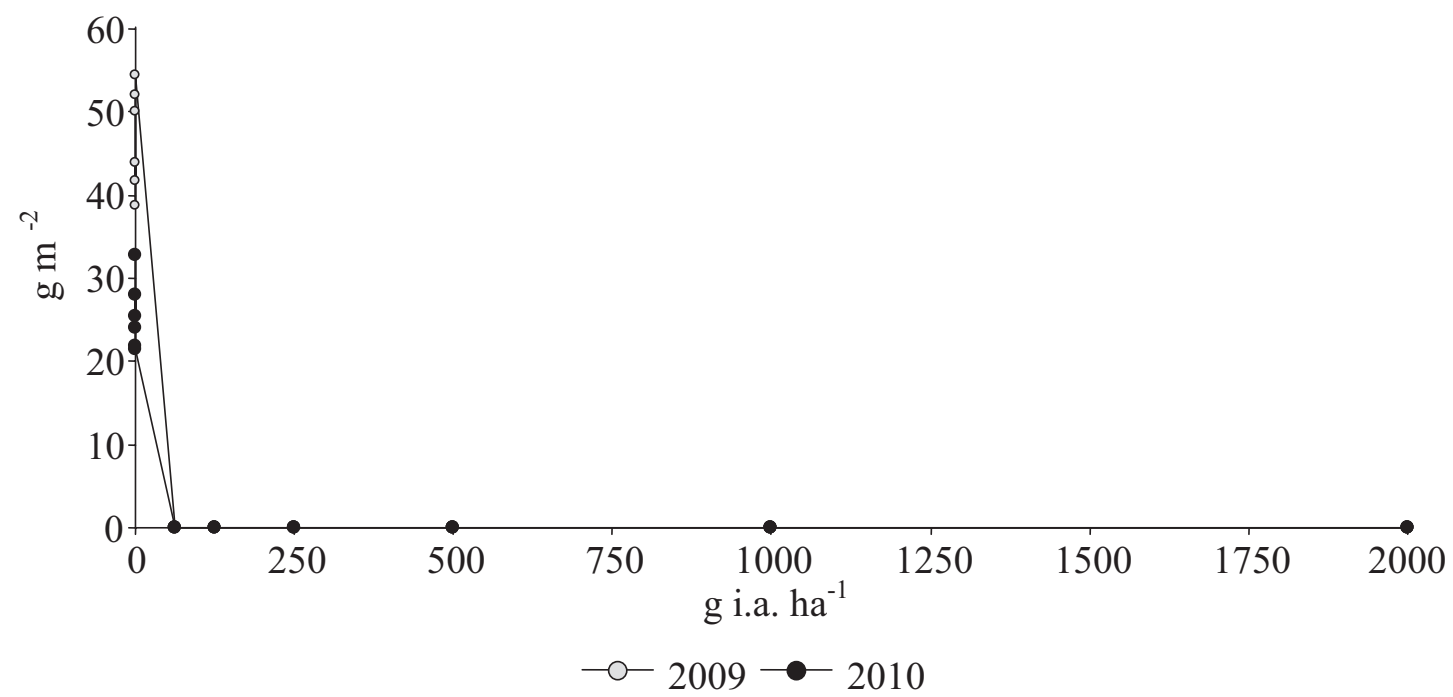

Figura 2. Efecto de diferentes dosis de 2,4-DB (g a.i. ha-1) sobre el peso seco (g.m²) de A. bicolor para 2009 y 2010.

tivo en el momento de aplicación (Lyon \& Wilson, 1986; Snipes et al., 1991; Wehtje et al., 1993; York et al., 1993; Hicks et al., 1998).

Al incrementarse los días después de la aplicación la fitotoxicidad se acrecienta hasta llegar a un máximo para todas las dosis (Tabla 2).

\section{Control de malezas en A. bicolor}

Las malezas latifoliadas relevadas asociadas a A. bicolor fueron en su mayoría de ciclo anual. Las especies más abundantes, frecuentes y de mayor grado de cobertura fueron Lamium amplexicaule, Veronica didyma, Bowlesia incana y Sonchus oleraceus, en agosto de 2009; Heterosperma ovatifolium, Portulaca oleracea, Taraxacum officinale, $L$. amplexicaule, $B$. incanay Bidens plilosa, en marzo 2010. Estas mismas especies fueron encontradas por Vitta et al. (1999), Bianco et al. (2000), Papa (2005) y Ferri et al. (2009) tanto en situaciones de cultivo como en relictos naturales de la zona de estudio (Tablas 3 y 4). Las malezas fueron bien controladas en su mayoría por ambos herbicidas; escaparon al control, en un 20\%, Lamium amplexicaule, Veronica didyma y Taraxacum officinale.

Cuando se analizó porcentaje de control de malezas asociadas a $A$. bicolor la interacción triple año $x$ herbicida $x$ dosis no fue significativa, con un $p=0,4203$. Los efectos de los factores herbicida
( $p=0,0021)$ y concentración $(p<0,0001)$ fueron significativos, mientras que la interacción año $x$ herbicida $y$ año $x$ dosis no lo fueron $(p=0,05)$.

El control con imazetapir a partir de dosis $1 \mathrm{X}$ (100 g i.a. ha ${ }^{-1}$ ) fue $80 \%$ (Figura 3). Verzignassi et al. (2005) en Stylosantes capitata y S. macrocephala muestra resultados prometedores para el control de malezas con imazetapir $100 \mathrm{~g}$ i.a. ha ${ }^{-1}$ en postemergencia. Rivas Pantoja et al. (2009) determinaron que la aplicación en postemergencia de imazetapir mejora la producción de materia seca en pasturas consociadas con Leucaena leucocephala (Lam.) de Wit debido al eficaz control de malezas. Ríos y Pérez (1990) y Castro (1993) coinciden con este estudio en los buenos resultados de control de malezas latifoliadas con imazetapir en Phaseolus vulgaris a dosis de 75 a $150 \mathrm{~g}$ i.a. ha-1.

El control con 2,4-DB fue adecuado con dosis menores de $1 \mathrm{X}$ (Figura 4), aunque como se mencionó es altamente fitotóxico sobre $A$. bicolor aun a dosis muy bajas, por lo que no es recomendable.

\section{CONCLUSIONES}

Los resultados de fitotoxicidad y control de malezas en $A$. bicolor muestran que es posible controlar la mayoría de las malezas de la región de estudio con imazetapir a la dosis de $100 \mathrm{~g}$ i.a. ha-1. Esta dosis del herbicida combina baja fitotoxicidad y un buen control de malezas. Si bien con 2,4-DB 
Tabla 3. Relevamiento de malezas asociadas a A. bicolor 30 días antes de la aplicación en 2009. Referencias: muestreos=210, individuos= 1389. $A=$ anual, $B=$ bianual, $P=$ perenne; $P=$ primavera, $v=$ verano; $O=$ otoño, $i=$ invernal. * $A$-vegeta todo el año y fructifica en $p-v$.

\begin{tabular}{|c|c|c|c|c|c|c|c|c|c|c|}
\hline Listado de malezas & $\begin{array}{l}\frac{\sigma}{0} \\
\frac{\sigma}{\sigma} \\
\frac{0}{0} \\
\frac{5}{J} \\
\frac{0}{4}\end{array}$ & $\begin{array}{l}\frac{\pi}{J} \\
\frac{1}{\Phi} \\
\stackrel{0}{0} \\
0\end{array}$ & $\begin{array}{l}\frac{\dot{0}}{0} \\
\frac{D}{0} \\
\frac{0}{0} \\
0 \\
\frac{0}{0} \\
0 \\
0 \\
0 \\
0 \\
0 \\
0 \\
0 \\
0\end{array}$ & 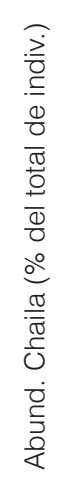 & 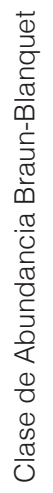 & 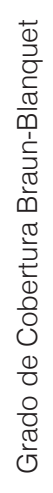 & 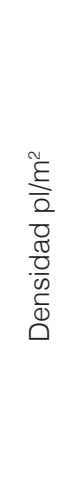 & 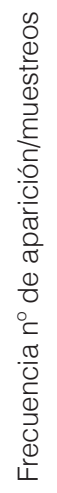 & $\begin{array}{l}\stackrel{\widetilde{\sigma}}{\overline{\bar{E}}} \\
\stackrel{\widetilde{\varpi}}{\leftarrow}\end{array}$ & 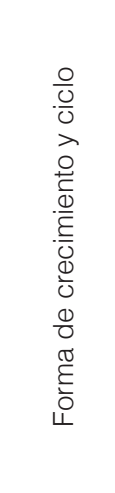 \\
\hline Lamium amplexicaule L. & 283 & 1155 & 4,1 & 20,4 & 5 & 2 & 11,3 & 61 & Lamiáceas & $\mathrm{A} / \mathrm{O}-\mathrm{i}$ \\
\hline Cyperus rotundus L. & 210 & 109 & 0,5 & 15,1 & 4 & 1 & 8,4 & 17 & Ciperáceas & $P / p-v$ \\
\hline Stipa brachychaeta Godr. & 185 & 347 & 1,9 & 13,3 & 3 & 2 & 7,4 & 38 & Poáceas & P/o-i \\
\hline Bowlesia incana Ruiz \& Pav. & 134 & 814 & 6,1 & 9,7 & 3 & 2 & 5,4 & 39 & Apiáceas & $\mathrm{A} / \mathrm{O}-\mathrm{i}$ \\
\hline Veronica didyma Ten. & 121 & 848 & 7,0 & 8,7 & 2 & 2 & 4,8 & 44 & Escrofulariáceas & A/o-i \\
\hline Sonchus oleraceus L. & 101 & 515 & 5,1 & 7,3 & 2 & 2 & 4,0 & 32 & Asteráceas & A/o-i \\
\hline Bromus catharticus Vahl & 91 & 582 & 6,4 & 6,6 & 2 & 2 & 3,6 & 23 & Poáceas & A/o-i \\
\hline Chenopodium album L. & 54 & 327 & 6,1 & 3,9 & 1 & 2 & 2,2 & 21 & Quenopodiáceas & * \\
\hline Sonchus asper (L.) Hill & 31 & 137 & 4,4 & 2,2 & 1 & 1 & 1,2 & 9 & Asteráceas & A/o-i \\
\hline Baccharis pingraea DC. & 30 & 186 & 6,2 & 2,2 & 1 & 1 & 1,2 & 14 & Asteráceas & A/o-i \\
\hline Rumex crispus L. & 27 & 263 & 9,7 & 1,9 & 1 & 1 & 1,1 & 8 & Poligonáceas & P/o-i \\
\hline Gamochaeta filanginea (DC.) & 27 & 99 & 3,7 & 1,9 & 1 & 1 & 1,1 & 12 & Asteráceas & P/o-i \\
\hline Taraxacum officinale Weber ex F. H. Wigg & 25 & 210 & 8,4 & 1,8 & 1 & 1 & 1,0 & 9 & Asteráceas & P/o-i \\
\hline Conyza bonariensis (L.) & 20 & 104 & 5,2 & 1,4 & 1 & 1 & 0,8 & 6 & Asteráceas & * \\
\hline Brassica rapa L. & 13 & 173 & 13,3 & 0,9 & 1 & 1 & 0,5 & 6 & Brasicáceas & A/o-i \\
\hline Oenotera indecora Cambess. & 9 & 39 & 4,3 & 0,7 & 1 & 1 & 0,4 & 5 & Onagráceas & $A / p-v$ \\
\hline Cotula australis (Sieber ex Spreng.) Hook. F. & 4 & 35 & 8,8 & 0,3 & 1 & 1 & 0,2 & 2 & Asteráceas & A/o-i \\
\hline Descurainia argentina O. E. Schulz & 4 & 25 & 6,3 & 0,3 & 1 & 1 & 0,2 & 2 & Brasicáceas & A/o-i \\
\hline Linaria canadensis (L.) Dum. Cours. & 3 & 40 & 13,3 & 0,2 & 1 & 1 & 0,1 & 2 & Escrofulariáceas & A/o-i \\
\hline Hirschfeldia incana (L.) Lagr.-Fossat & 3 & 25 & 8,3 & 0,2 & 1 & 1 & 0,1 & 1 & Brasicáceas & $A-B / o-p$ \\
\hline Melilotus albus Desr. & 3 & 14 & 4,7 & 0,2 & 1 & 1 & 0,1 & 2 & Fabáceas & $A-B / p-v$ \\
\hline Eleusine tristachya (Lam.) Lam. & 3 & 8 & 2,7 & 0,2 & 1 & 1 & 0,1 & 1 & Poáceas & $A / p-V$ \\
\hline Poa annua L. & 2 & 10 & 5,0 & 0,1 & 1 & 1 & 0,1 & 1 & Poáceas & A/o-i \\
\hline Verbascum virgatum stokes ex With. & 2 & 9 & 4,5 & 0,1 & 1 & 1 & 0,1 & 1 & Escrofulariáceas & $A-B / 0-i$ \\
\hline Oenothera laciniata Hill & 2 & 6 & 3,0 & 0,1 & 1 & 1 & 0,1 & 1 & Onagráceas & $A / p-v$ \\
\hline Carduus tohermeri Weinm. & 1 & 20 & 20,0 & 0,1 & 1 & 1 & 0,0 & 1 & Asteráceas & A/o-i \\
\hline Cenchrus pauciflorus Benth. & 1 & 5 & 5,0 & 0,1 & 1 & 1 & 0,0 & 1 & Poáceas & $A / p-v$ \\
\hline
\end{tabular}

se logra buen control de malezas, el herbicida es extremadamente fitotóxico para $A$. bicolor aún a dosis menores a $250 \mathrm{~g}$ i.a. ha-1 por lo que no es recomendable su uso.

\section{BIBLIOGRAFÍA}

Aguilera, M.O.; D.F. Steinaker, M.R. Demaría y A.O. Ávila, 1998. Estados y transiciones de los pastizales Sorghastrum pellitum del área medanosa central de
San Luis, Argentina. Ecotrópicos 11(2):107-120.

Altman, J. and A.D. Rovira, 1989. Herbicide-pathogen interactions in soil-borne root diseases. Canadian Journal of Plant Pathology 11:166-172.

Artuzi, J.P. y R.L. Contiero, 2006. Herbicidas aplicados na soja e produtividade do milho em sucessão. Pesquisa Agropecuaria 41(7):1119-1123.

Autrán, V. A. and E. C. Puricelli, 2010. Herbicide effect on Adesmia bicolor, a forage leguminous of marginal areas. Biocell 34(1):A27-A78. 
Tabla 4. Relevamiento de malezas asociadas a A. bicolor 30 días antes de la aplicación en 2010. Referencias: muestreos $=210$, individuos $=1541 . A=$ anual, $B=$ bianual, $P=$ perenne; $p=$ primavera, $v=$ verano; $\mathrm{o}=$ otoño, $i=$ invernal. ${ }^{*} \mathrm{~A}$-vegeta todo el año y fructifica en $\mathrm{p}$-v.

\begin{tabular}{|c|c|c|c|c|c|c|c|c|c|c|}
\hline Listado de malezas & $\begin{array}{l}\frac{\sigma}{0} \\
\frac{C}{\sigma} \\
\frac{\sigma}{O} \\
\frac{C}{J} \\
\frac{0}{<}\end{array}$ & 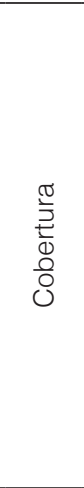 & 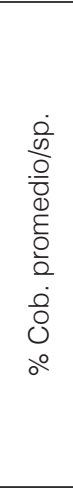 & 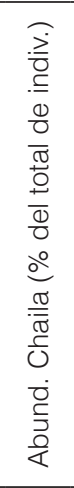 & 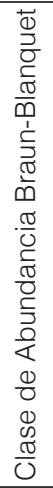 & 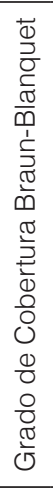 & $\begin{array}{l}\text { है } \\
\frac{\text { Q }}{0} \\
\overline{0} \\
\frac{0}{0} \\
\frac{0}{\omega} \\
\frac{C}{1} \\
0\end{array}$ & 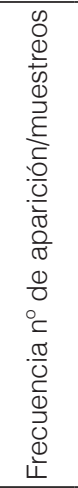 & 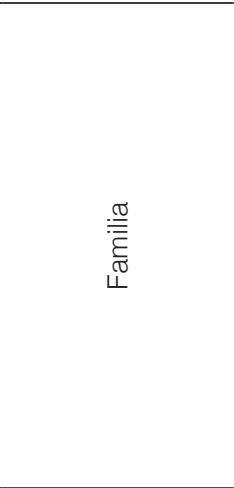 & 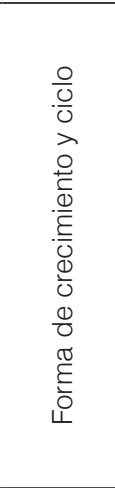 \\
\hline Heterosperma ovatifolium Cav. & 378 & 3565 & 44 & 19,7 & 5 & 3 & 15,1 & 90 & Asteráceas & $A / p-v$ \\
\hline Portulaca oleracea L. & 287 & 480 & 12,6 & 14,9 & 4 & 2 & 11,5 & 68,3 & Portulacáceas & $A / p-V$ \\
\hline Taraxacum officinale Weber. Ex F. H. Wigg & 157 & 1871 & 19,1 & 8,2 & 2 & 2 & 6,3 & 37,4 & Asteráceas & $\mathrm{P} / \mathrm{O}-\mathrm{i}$ \\
\hline Lamium amplexicaule L. & 110 & 45 & 5,6 & 5,7 & 3 & 2 & 4,4 & 26,2 & Lamiáceas & A/o-i \\
\hline Bowlesia incana Ruiz \& Pav. & 109 & 42 & 6 & 5,7 & 3 & 2 & 4,3 & 26 & Apiáceas & A/o-i \\
\hline Bidens pilosa L. & 90 & 920 & 31,7 & 4,7 & 1 & 3 & 3,6 & 21,4 & Asteráceas & $A / p-V$ \\
\hline Chenopodium album L. & 82 & 725 & 16,5 & 4,2 & 2 & 2 & 3,3 & 19,5 & Quenopodiáceas & * \\
\hline Chenopodium pumilo R. Br. & 82 & 340 & 11 & 4,2 & 1 & 2 & 3,3 & 19,5 & Quenopodiáceas & $A / p-v$ \\
\hline Amaranthus quitensis Kunth. & 41 & 505 & 16,3 & 2,1 & 1 & 2 & 1,6 & 9,8 & Amarantáceas & $A / p-v$ \\
\hline Conyza bonariensis (L.) & 39 & 465 & 18,6 & 2,0 & 1 & 2 & 1,5 & 9,3 & Asteráceas & * \\
\hline Dichondra microcalyx Meisn & 26 & 35 & 17,5 & 1,3 & 1 & 2 & 1,0 & 6,2 & Convolvuláceas & $P / p-v$ \\
\hline Sonchus oleraceus L. & 23 & 135 & 6,7 & 1,2 & 1 & 2 & 0,9 & 5,5 & Asteráceas & A/o-i \\
\hline Galinsoga parviflora Cav. & 22 & 220 & 16,9 & 1,1 & 1 & 2 & 0,9 & 5,2 & Asteráceas & $A / p-V$ \\
\hline $\begin{array}{l}\text { Euphorbia hirta L. var. Ophtalmica (Pers.) } \\
\text { Allen \& Irgang }\end{array}$ & 14 & 175 & 15,9 & 0,7 & 1 & 2 & 0,5 & 3,3 & Euforbiáceas & $A / p-V$ \\
\hline Rumex crispus L. & 14 & 90 & 6,4 & 0,7 & 1 & 2 & 0,5 & 3,3 & Poligonáceas & P/O-i \\
\hline Polygonum aviculare L. & 12 & 300 & 75 & 0,6 & 1 & 5 & 0,5 & 2,9 & Poligonáceas & A/o-i \\
\hline Bidens subalternans DC. & 11 & 175 & 17,5 & 0,5 & 1 & 2 & 0,4 & 2,6 & Asteráceas & $A / p-V$ \\
\hline Gnaphalium gaudichaudianum DC. & 9 & 130 & 14,4 & 0,4 & 1 & 2 & 0,3 & 2,1 & Asteráceas & A/o-i \\
\hline Verbena litoralis Kunth. & 7 & 100 & 14,3 & 0,3 & 1 & 2 & 0,3 & 1,7 & Verbenáceas & $P / p-V$ \\
\hline Ulmus pumila L. & 7 & 65 & 21,7 & 0,3 & 1 & 2 & 0,3 & 1,7 & Ulmáceas & $P / p-v$ \\
\hline Euphorbia dentata Michx. & 5 & 65 & 13 & 0,2 & 1 & 2 & 0,2 & 1,2 & Euforbiáceas & $A / p-V$ \\
\hline Carduus acantoides L. & 4 & 150 & 37,5 & 0,2 & 1 & 3 & 0,2 & 1 & Asteráceas & A/O-i \\
\hline Abutilon pauciflorum A. St.- Hill. & 4 & 45 & 11,3 & 0,2 & 1 & 2 & 0,2 & 1 & Malváceas & $P / p-v$ \\
\hline Melilotus albus Desr. & 3 & 15 & 5 & 0,1 & 1 & 2 & 0,1 & 0,7 & Fabáceas & $A-B / p-v$ \\
\hline Mollugo verticillata $\mathrm{L}$. & 2 & 30 & 15 & 0,1 & 1 & 2 & 0,1 & 0,5 & Molugináceas & $\mathrm{A} / \mathrm{p}-\mathrm{V}$ \\
\hline Hirschfeldia incana (L.) Lagr.- Fossat & 2 & 25 & 12,5 & 0,1 & 1 & 2 & 0,1 & 0,5 & Brasicáceas & A-B/o-p \\
\hline Malvastrum coromandelianum L. Garcke & 1 & 15 & 15 & 0,05 & 1 & 2 & 0,1 & 0,2 & Malváceas & $\mathrm{P} / \mathrm{p}-\mathrm{V}$ \\
\hline
\end{tabular}

Bianco, C. 2002. Growth forms, taxonomy, distribution, and uses of the Adesmia Species (Leguminosae) in Central Argentina. Cramer. Stuttgart, Germany. 156 pp.

Bianco, C.; C. Nuñez y T. Kraus, 2000. Identificación de frutos y semillas de las principales malezas del centro de Argentina. Río Cuarto. Fundación Universidad Nacional de Río Cuarto. 142 pp.

Cano O. y E. López, 1996. Control preemergente y pos- temergente de malezas en frijol, de humedad residual en Veracruz, México. Agronomía Mesoamericana $7(2): 42-49$.

Canobas, V.C.; J.A. Bongiovanni, A.H. Beneitez, C.M. Ferri, N. P. Stritzler y H. J. Petruzzi, 2006. Producción de forraje en pasturas puras y en mezclas de alfalfa, festuca alta y pasto Wilman. EEA Anguil. INTA. Anguil. AR. Investigación en producción animal Región subhúmeda y semiárida pampeana, Boletín de divulga- 


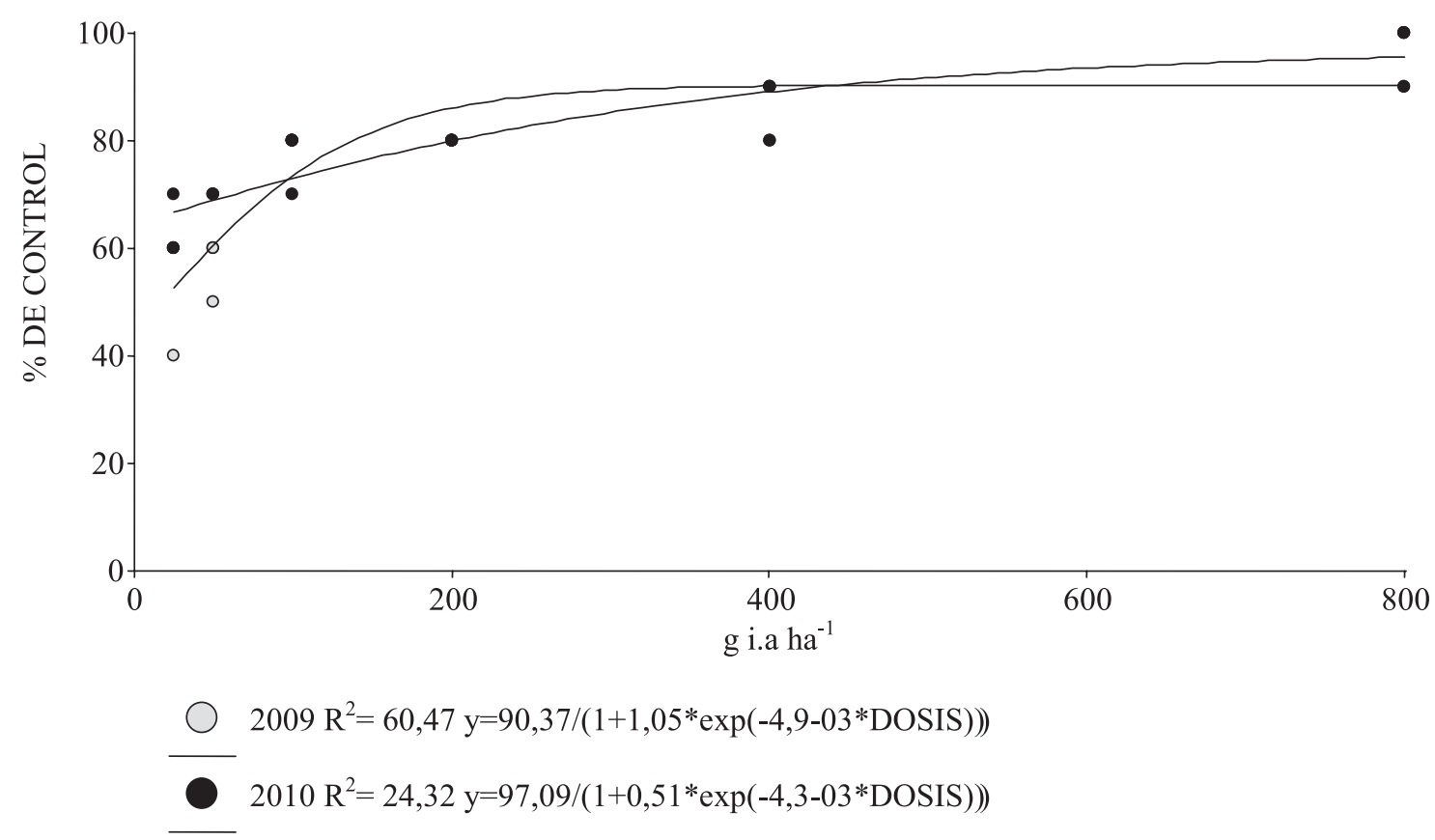

Figura 3. Efecto de diferentes dosis de imazetapir (g a.i. ha-1 ${ }^{-1}$ sobre $(\%)$ de control de malezas promedio 2009 y 2010. 2009: $R^{2}=60,47$ y=90,37/(1+1,05*exp $\left(-4,9^{-03 *}\right.$ DOSIS) $) 2010: R^{2}=24,32$ y=97,09/ $\left(1+0,51^{\star} \exp \left(-4,3^{-03 *} \mathrm{DOSIS}\right)\right)$

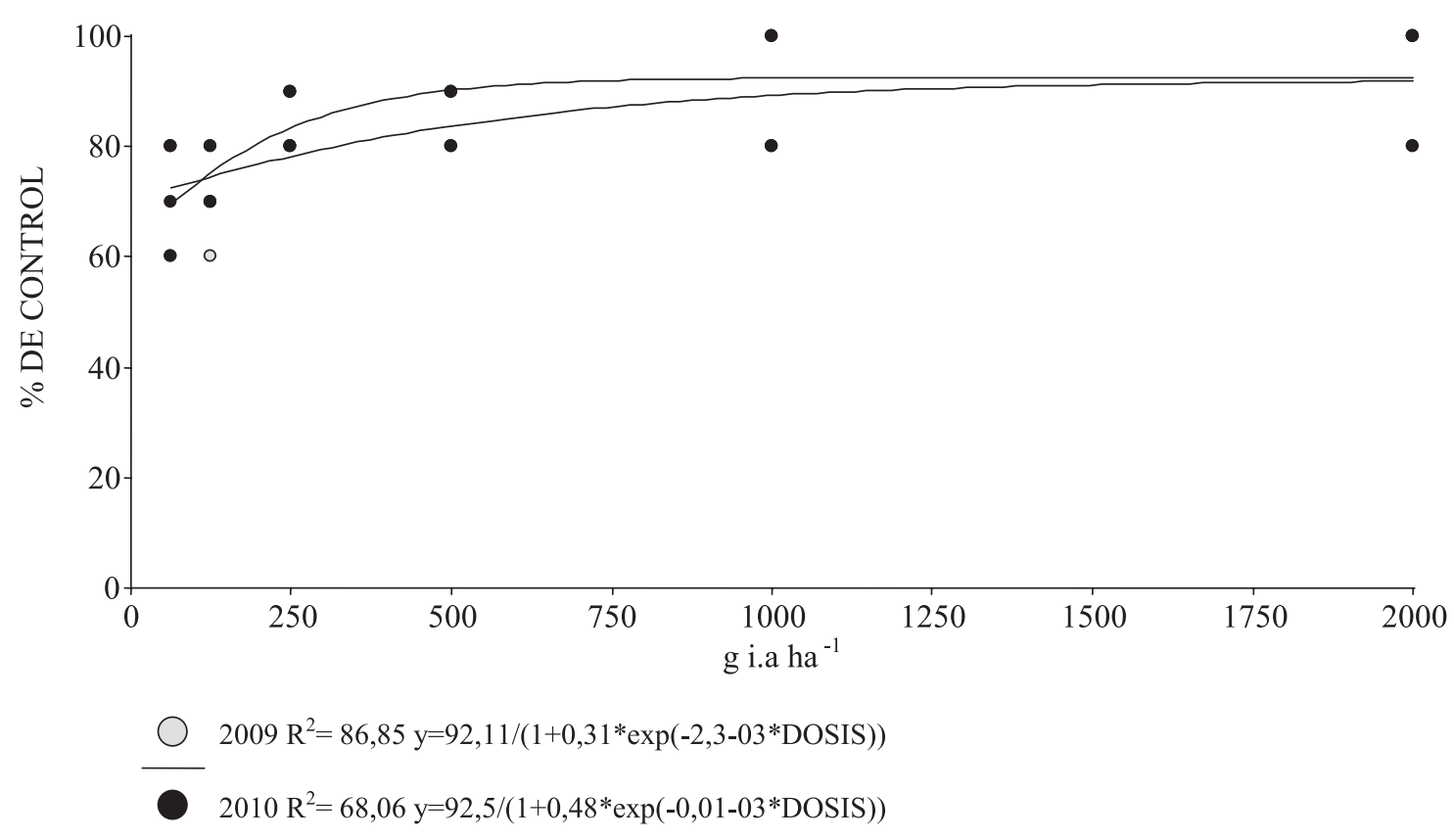

Figura 4. Efecto de diferentes dosis de 2,4-DB ( $\mathrm{g}$ a.i. ha $\left.{ }^{-1}\right)$ sobre el porcentaje (\%) de control de malezas promedio 2009 y 2010. 2009: $R^{2}=86,85$ y=92,11/(1+0,31*exp(-2,3-03*DOSIS))2010: $R^{2}=68,06$ y=92,5/ $\left(1+0,48^{*} \exp \left(-0,01^{-03 *} \mathrm{DOSIS}\right)\right)$ 
ción técnica EEA Anguil Nº 90.

Carson, M.L.; W.E. Arnold and P.E. Todt, 1991. Predisposition of soybean seedling to Fusarium root rot with trifluralin. Plant Disease 75:342-347.

Castillo Gallegosa, E.; B. Valles de la Moraa, L. Mannetjeb y A. Aluja Schunemannc, 2005. Efecto de introducir Arachis pintoi sobre variables del suelo de pasturas de grama nativa del trópico húmedo mexicano. Técnica Pecuaria 43(2):287-295

Castro, M.E. 1993. Control químico de cadillo Xanthium strumarium L. en frijol en la región lagunera. In: Memoria XIV Congreso Nacional de la Ciencia en la Maleza, Puerto Vallarta, Jalisco. México. 56 pp.

Ceballos, R.; G. Palma, H. Brevis, F. Ortega and A. Quiroz, 2005. Effect of five postemergence herbicides on red clover shoot and root growth in greenhouse studies. Phytoprotection 85:153-160.

Chaila, S., 1986. Métodos de evaluación de malezas para estudios de población y de control. Revista de la Asociación Argentina para el control de Malezas. ASAM 14(2):79.

Coll, J. y A. Zarza, 1992. Leguminosas nativas promisorias. Trébol polimorfo y babosita. Boletín de Divulgación INIA (Uruguay) 22:19 pp.

Conrad J.D. y J.F. Stritzke, 1980. Response of Arrowleaf Clover to Postemergence Herbicides. Agronomy Journal 72:670-672.

Cudney, D.; S.B. Orloff y C. Adams, 1993. Improving weed control with 2,4 DB amine in seedling alfalfa (Medicago sativa). Weed Technology 7:465-470.

Darwent, A.L.; D. Cole and N. Malik, 1997. Imazethapyr, alone or with other herbicides for weed control during alfalfa (Medicago sativa) establishment. Weed Technology 11(2):346-353.

Demaría, M.R.; I. Aguado Suárez y D.F. Steinaker, 2008. Reemplazo y fragmentación de pastizales pampeanos semiáridos en San Luis, Argentina. Ecología Austral 18:55-70.

Di Rienzo, J.; F. Casanoves, M. Balzarini, L. Gonzalez, M. Tablada, C. Robledo, 2010. InfoStat, versión 2010. Grupo InfoStat, F.C.A. (UNC) Córdoba, Argentina.

Dissanayake, N.; J.W. Hoy and J.L. Griffin, 1998. Herbicide effects on sugarcane growth, Pythium root rot, and Pythium arrhenomanes. Phythopathology 88:530-535.

Dodd, M.B. and S.J. Orr, 1995. Seasonal growth, phosphate response, and drought tolerance of 11 perennial legume species grown in a hill-country soil. New Zealand Journal of Agricultural Research 38(1):7-20.

Eberlein, C.V. and M.J. Guttieri, 1994. Potato (Solanum tuberosum) response to simulated drift of imidazolinone herbicides. Weed Science 42:70-75.

Ferri, R.; M. Ceballos, N. Vischi, E. Heredia y A. Ogge- ro, 2009. Banco de semillas de un relicto de Espinal (Córdoba, Argentina). Iheringia, Série Botánica. Porto Alegre. 64(1):93-100.

Hess, F.D., 1993. Herbicide effects on plant structure, physiology and biochemistry. In: Pesticide Interactions in Crop Production Beneficial and Deleterious Effects. J. Altman. CRC Press, London. 579 pp.

Hicks, T.V.; G.R. Wehtje and T.L. Grey, 1998. The interaction of pyridate and 2,4-DB in peanut (Arachis hypogaea), Florida beggarweed (Desmodium tortuosum), and sicklepod (Senna obtusifolia). Weed Science 46:284-288.

Istilart, C., 2003. Malezas en pasturas de leguminosas consociadas con gramíneas forrajeras. Producción Bovina de Carne. Disponible en <http://www.inta.gov. ar/barrow/info/documentos/ganaderia/carpeta2002/ malezas> Consultada el 16/03/2011.

Lyon, D. and R. Wilson, 1986. Sensitivity of fieldbeans (Phaseolus vulgaris) to reduced rates of 2,4-D and dicamba. Weed Science 34(6): 953-956.

Ovalle, C.M.; A.L. del Pozo, S.S. Arredondo y J. Chavarría, 2005. Crecimiento y producción de nuevas leguminosas forrajeras anuales en la zona mediterránea de Chile. I. (Comportamiento de las especies en la precordillera andina). Agricultura Técnica 65(1):35-47.

Papa, J.C., 2005. Detección de especies de malezas de importancia emergente en el centro-sur de la provincia de Santa Fe. Soja. Para mejorar la producción. EEA Oliveros. INTA. 30:142-146.

Peregrine, E. and F. Norris, 1988. Environmental modification of seedling alfalfa, Medicago sativa, Tolerance to Bromoxinil. Weed Science 36:671-677.

Pinzón, B.R.; P.J. Argel y R. Montenegro, 1985. Control de malezas en el establecimiento de Kudzu tropical. Pasturas Tropicales 11(1):7-12.

Pinzón, B.R.; P.J. Argel y R. Montenegro, 1989. Selectividad de herbicidas y control de malezas en Centrosema macrocarpum. Pasturas Tropicales 7(2):6-8.

Rebuffo, M.; M. Bemhaja and D.F. Risso, 2006. Utilization of forage legumes in pastoral systems: state of art in Uruguay. Lotus Newsletter 36:2-33.

Renzi, J.P., 2007. Selectividad de herbicidas aplicados en postmergencia en Vicia villosa Roth. y Avena sativa L. Revista Argentina de Producción Animal 27(1): 150-151.

Renzi, J.P.; H. Boscardín y R. Agamennoni, 2006. Fitotoxicidad de herbicidas aplicados en postemergencia en Vicia villosa Roth. consociada con Avena sativa L. Revista Argentina de Producción Animal 26(1):63.

Rios, T.A. y M.L.A. Perez, 1990. Control químico postemergente de maleza en frijol. In: XI Congreso Nacional de Ciencia de la Maleza. México. 47 pp.

Rivas Pantoja, F.; J. Castillo Huchim y L. Ortega Reyes, 
2009. Selectividad de herbicidas y control de malezas para establecer una asociación Brachiaria brizanthaLeucaena leucocephala. Técnica Pecuaria México 47:339-355.

Rojas Hernández, S.; J. Olivares Pérez, R. Jiménez Guillén y E. Hernández Castro, 2005. Manejo de praderas asociadas de gramíneas y leguminosas para pastoreo en el trópico. Revista Electrónica de Veterinaria REDVET $®$ 6(5):1695-7504.

Sanabria V.; M. Damelys, U. Manrique, M. Rodríguez, A. Gil y P.J. Argel, 1995. Siembra de leguminosas en un pastizal establecido de Brachiaria decumbens. Zootecnia Tropical. Venezuela 13(2):245-260.

Scheffer-Basso, S.M.; C.M. Carneiro y M. Voss, 2000. Nodulação e fixação biológica de nitrogênio em Adesmia araujoi Burk. Revista Brasileira de Agrociência 6(1):16-18.

Scheffer-Basso, S.M. ; M. Voss y A.V. Ávila Jacques, 2001. Nodulação e fijação biológica de nitrogênio de Adesmia latifolia e Lotus corniculatus em vasos de Leonard. Revista Brasileira de Zootecnia 30(3):1-12.

Scheffer-Basso, S.M.; M.C. Vendrúsculo, K. Baréa, R. Cácio Benincá, R. Lubenow and D. Cecchetti, 2002. (Adesmia, Lotus, Trifolium) in mixture with tall fescue. Response of legumes. Revista Brasileira de Zootecnia 31:2197-2203.

Seefeldt S.; J. E. Jensen and E. P. Fuerst, 1995. Log-Logistic analysis of herbicide dose-response relationships. Weed Technology 9(2): 218-227.

Smiley, R.W. and D.E. Wilkins, 1992. Impact of sulfonylurea herbicides on Rhizoctonia root rot, growth and yield of winter wheat. Plant Disease 76:399-404.

Snipes C. E.; J. E. Streetand and T. C. Mueller, 1991. Cotton (Gossypium hirsutum) response to simulated triclopyr drift. Weed Technology 5:493-498.

Tonks, D.; S. Larry; Y. Jeffrery and B.L. Webb, 1991. Response of seedling alfalfa (Medicago sativa) to four postemergence herbicides. Weed Technology 5:736738.
Traverso, J.; H. Troiani y F. Babinec, 2005. Colección y conservación de las especies forrajeras nativas y naturalizadas de la provincia de La Pampa. Publicación Técnica INTA N 63. 20 pp.

Veneciano, J.H.; C.A. Frasinelli, T. Kraus y C.A. Bianco, 2005. Domesticación de especies forrajeras. UNRCINTA. 60 pp.

Verzignassi J.R.; F. de Assis Rolim Pereira, C. Dornelas Fernandes y J. Batista Vida, 2005. Selectividad de herbicidas a Stylosanthes capitata E A S. macrocephala. Revista Brasileira de Herbicidas 3:59-67.

Vileta, D.; L. Bianco, M. Grosso and R. Malpassi, 2010. Biological nitrogen fixation by Adesmia bicolor and $A$. macrostachya, potential forage species for arid and semi-arid environments. Interciencia 35(02):120-125.

Vitta, J.; D. Faccini, L. Nisensohn, E. Puricelli, D. Tuesca y E. Leguizamón, 1999. Las malezas en la región sojera núcleo Argentina: Situación actual y perspectivas. Dow AgroSciences. Rosario. 47 pp.

Wehtje, G.; J.W. Wilcut and J.A. McGuire, 1993. Absorption, translocation, and phytotoxicity of chlorimuron and 2,4-DB mixtures in peanut (Arachis hypogaea) and selected weed species. Weed Science 41:347352.

Wilson, R.G Jr., 1981. Weed control in established dryland alfalfa (Medicago sativa). Weed Science 29:615-618.

York, A.C.; J.W. Wilcut and W.J. Grichar, 1993. Interaction of 2,4-DB with postemergence graminicides. Peanut Science 20:57-61

Young, B.G.; J.M. Young, J.L. Mathews, M.D. Owens, I.A. Zelaya, R.G. Hartzler, L.M. Wax, K.W. Rorem and G.A. Bollero. 2003. Soybean development and yield as affected by three postemergence herbicides. Agronomy Journal 95:1152-1156.

Zalazar, M.; G. Funes y M.P. Venier, 2009. Factores que afectan la germinación de Justicia squarrosa Griseb, forrajera nativa de la región chaqueña de la Argentina. Agriscientia 16(1):1-6. 